\title{
BEAM MEASUREMENT SYSTEMS FOR THE CERN ANTIPROTON DECELERATOR (AD)
}

\author{
V.Chohan, M. Angoletta, M. Ludwig, O. Marqversen, P. Odier, F. Pedersen, G.Tranquille, U.Raich, \\ L. Soby, CERN, Geneva, Switzerland \\ T. Spickermann, LANL, Los Alamos, USA
}

\begin{abstract}
The new, low-energy antiproton physics facility at CERN has been successfully commissioned and has been delivering decelerated antiprotons at $100 \mathrm{MeV} / \mathrm{c}$ since July 2000. The $\mathrm{AD}$ consists of one ring where the 3.5 $\mathrm{GeV} / \mathrm{c}$ antiprotons produced from a production target are injected, rf manipulated, stochastically cooled, decelerated (with further stages involving additional stochastic and electron cooling and rf manipulation) and extracted at $100 \mathrm{MeV} / \mathrm{c}$. While proton test beams of sufficient intensity could be used for certain procedures in AD commissioning, this was not possible for setting-up and routine operation. Hence, special diagnostics systems had to be developed to obtain the beam and accelerator characteristics using the weak antiproton beams of a few $10^{7}$ particles at all momenta from $3.5 \mathrm{GeV} / \mathrm{c}$ down to 100 $\mathrm{MeV} / \mathrm{c}$. These include systems for position measurement, intensity, beam size measurements using transverse aperture limiters and scintillators and Schottky-based tools. This paper gives an overall view of these systems and their usage.
\end{abstract}

\section{INTRODUCTION}

The AD provides antiprotons at low-energy for physics in a much simplified manner than the series of machines that were necessary in the past. Full details about the AD are well-documented [1], [2], [3] with the functioning best illustrated in Fig 1, which shows the essentials of the AD operation. Starting with $\sim 5 \times 10^{7}$ injected antiprotons in $190 \pi$ mm.mrad machine acceptance and $\pm 3 \%$ momentum spread, the beam ejected is of the order of 2 to $3 \times 10^{7}$ antiprotons in a single bunch of $230-400 \mathrm{~ns}$ with a momentum spread of $\sim 0.2 \%$ and transverse emittances < $1.5 \pi \mathrm{mm} . \mathrm{mrad}$. The different beam cooling systems in the deceleration process not only compensate for the adiabatic beam blow-up but also cool the beam even further. Great emphasis had to be put on certain beam measurement systems, e.g., monitoring of the beam position, size and intensity through the $\mathrm{AD}$ cycle with the beam intensity of $\sim 10^{7}$ particles, corresponding to $\sim 260$ $\mathrm{nA}$ at $100 \mathrm{MeV} / \mathrm{c}$. Hence, greater exposure has been given to these particular systems in this paper, with other installed systems briefly mentioned at the end.

\section{POSITION MEASUREMENTS}

During deceleration, several plateaux are introduced to permit different stochastic or electron cooling systems to act on the beam. It was desirable that at each intermediate energy level, the orbit should be measurable and if necessary, corrected.

The closed orbit measurement system [4] employs 59 electrostatic pick-ups (PU). The intensity range from $2 \times 10^{10}$ down to $10^{7}$ particles poses challenging demands on the dynamic range and noise of the head amplifier. A low-noise amplifier was developed, having an equivalent input noise of $0.6 \mathrm{nV} / \sqrt{\mathrm{Hz}}$, allowing beam positions to be measured to $\pm 0.5 \mathrm{~mm}$ with as few as $5 \times 10^{6}$ particles. Two different gains take care of the large dynamic range. After amplification and multiplexing, the PU signals are fed to a network analyser, where every measurement point corresponds to one PU. The network analyser is locked to the rf of the $\mathrm{AD}$, thus acting as a "tracking filter" instrument. An orbit measurement takes from 0.5 to $12 \mathrm{~s}$ depending on the IF-bandwidth of the network analyser, which is selected according to the beam intensity, and the precision required. At the end of the network analyser sweep, the data are read via a GPIB interface and treated by a real-time task running in a VME crate.

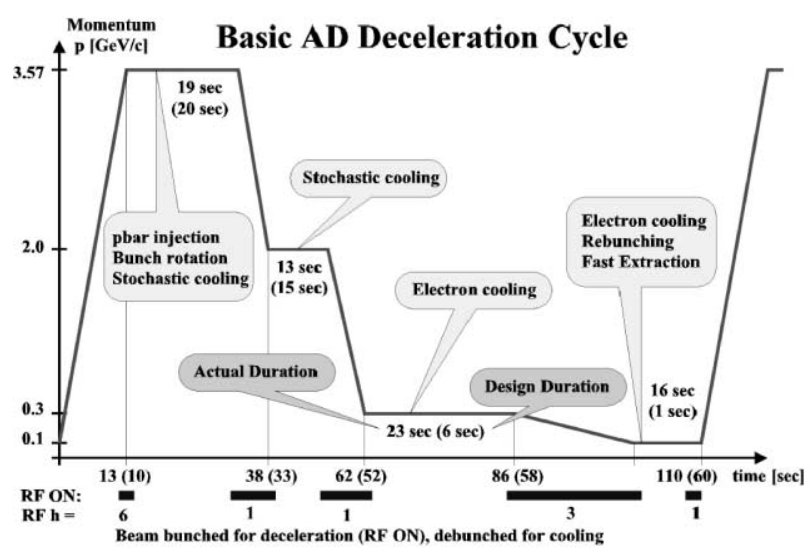

Figure 1: AD Cycle \& Plateaux with Processes

\section{INTENSITY MEASUREMENTS}

\subsection{Higher intensity $\left(\sim 10^{9}\right)$ circulating beams}

A dc Beam Transformer (DCBT) is used to measure the bunched or unbunched circulating beam current. The device has been particularly adapted to the AD needs, deceleration and control system. In order to provide a measurement of the beam charge through out the energy range, a so-called relativistic $B$ normaliser has been added. It is essentially an amplifier whose gain is proportional to $1 / \beta$; the value of $\beta$ is deduced from the 
value of the magnetic flux density in the bending magnets. The device resolution is typically $1.5 \mu \mathrm{A}$, which corresponds to a beam of $5.9 \times 10^{6}$ charges at injection ( $3.57 \mathrm{GeV} / \mathrm{c}, \beta=0.967)$; the resolution deteriorates due to deceleration and corresponds to $5.4 \times 10^{7}$ charges at ejection $(100 \mathrm{MeV} / \mathrm{c}, \beta=0.106)$. This value is adequate to measure the protons used in the setting-up mode (typical beam of $5.10^{9}$ charges) but is insufficient for the operational antiproton beams of $\sim 5.10^{7}$ charges. Hence, reasonable observations may be carried out only at high momenta, i.e. $3.57 \mathrm{GeV} / \mathrm{c}$ and $2 \mathrm{GeV} / \mathrm{c}$.

The DCBT values with sufficient proton beams are used to cross-calibrate the low intensity DRX-based measurement system.

\subsection{Low Intensity $\left(\sim 10^{7}\right)$ circulating beams}

An innovative system using a commercial Digital Receiver (DRX) VME board has been developed [5] to measure low intensity beams. The system also measures mean momentum and momentum spread. Signals coming from a wide-band $(0.02-30 \mathrm{MHz})$ ultra-low-noise AC beam transformer [6], acting as a longitudinal Schottky $\mathrm{PU}$, are conditioned, digitised and processed by the DRX, which hosts 8 Digital Down Converters (DDC) and one floating-point Digital Signal Processor (DSP), shown in Fig.2.

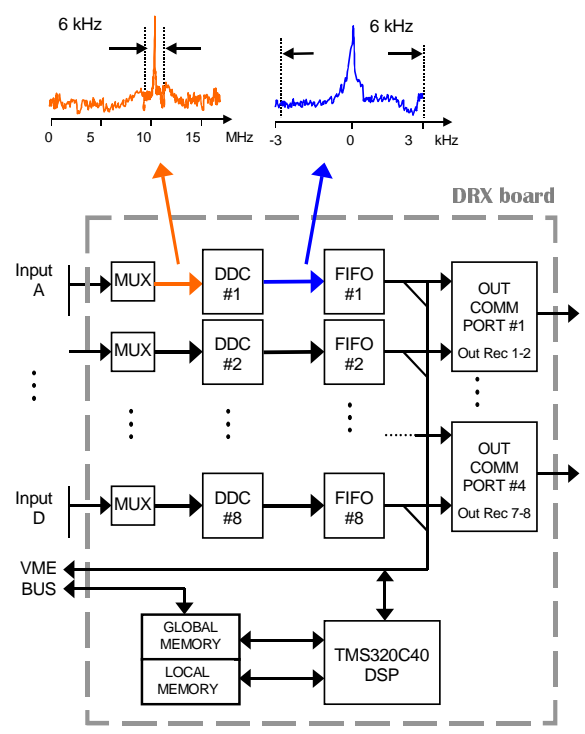

Figure 2: Simplified DRX flowchart

The DDCs initially process the signals in hardware, i.e., translate digitally the input signals down to dc and execute a zoom by digital filtering on the frequency window of interest. The DSP software provides the further processing stage. The actual signal processing depends on the beam state; for debunched beams, the intensity and other longitudinal beam properties are obtained by FFT-based spectral analysis of Schottky signals. For bunched beams, the intensity is obtained by measuring the amplitude of the fundamental and second harmonic of the rf. Post-DRX processing for calibration and further treatment is done in the real-time task, yielding the intensity values.

\section{BEAM SIZE EVALUATION}

A pair of horizontal and a pair of vertical scrapers, situated in a dispersion-free region, are used to measure the transverse amplitude distribution and thus the size of the beam. They consist of $1 \mathrm{~mm}$ thick tungsten blades that are moved into the beam (test proton beams or antiprotons) with stepping motors. Despite being destructive, this is the method of choice in the AD due to the simplicity of usage with low intensity antiproton beams. The scrapers also permit measurement of machine acceptances when coupled with a rf 'blow-up' system to excite the test beams and fill the aperture. Finally, the scrapers are a handy tool for tailoring the beam, i.e. collimating the beam to reduce its size or intensity. Protons that hit the scraper blade undergo multiple scattering and are eventually lost, while for antiprotons, annihilation takes place. Therefore, one measures a reduction in beam current. Downstream scintillators detect particles produced in the annihilation process between antiprotons and the blade.

The "classical" method is to move a blade into the beam in small steps and measure the remaining current after or the scintillator counts during, each step. This "slow" mode is useful to obtain a precise measurement of the centre of the beam. At very low intensity of the remaining beam, where neither the beam current nor the count rate can provide a significant signal, one can still use the Schottky signal to determine whether the entire beam has been eliminated. The other major advantage of the "slow" method is that it permits tailoring of the beam to a desired size or intensity. This has been extremely valuable during tests with protons, for example to test the effectiveness of stochastic and electron cooling.

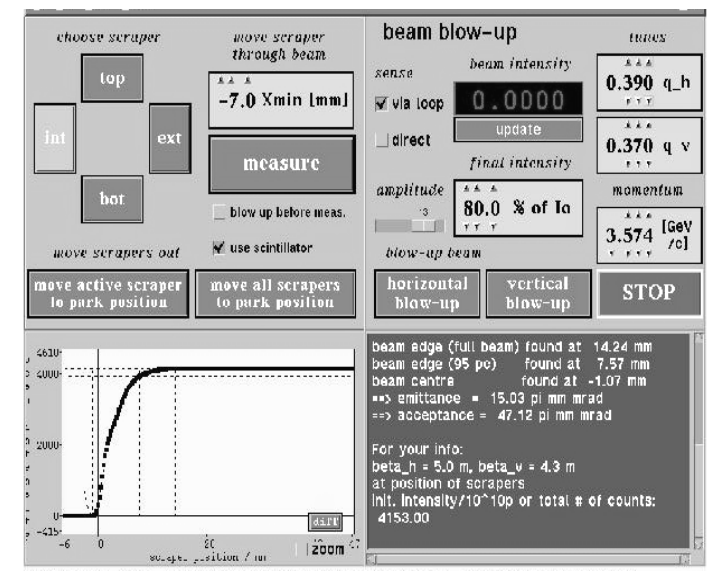

Figure 3: A typical Emittance Measurement Display

In the "fast" mode (Fig. 3), a blade is moved through the beam in one sweep with a constant speed of $25 \mathrm{~mm} / \mathrm{s}$ and the blade position acquired every $40 \mathrm{~ms}$, i.e. $1 \mathrm{~mm}$ spacing. The blade position and beam current are read and stored with their respective time tags. These time tags are correlated to obtain a curve of beam current as a function of the scraper position. If the scintillators are used, the scraper movement and the scintillator measurements are synchronised and the position and counts are read at the same time. The number of counts up to a certain blade 
position is subtracted from the total number of counts, yielding an amplitude distribution similar to the one for the beam current. Using the scintillators extends the measurement range to very low beam intensities, where the DCBT is not sensitive enough.

The beam current or the scintillator counts rate as a function of the scraper position may be differentiated to obtain the "betatron amplitude distribution". The function is plotted at the end of the measurement and numerical fits are applied to the positions of the edge and centre of the beam. The "95\%-edge", i.e., the scraper position where the remaining beam current is $95 \%$ of the initial current, is determined by interpolation. For the projected one-dimensional Gaussian distribution characterised by value $\sigma$, the emittance $\varepsilon$ is calculated as $\varepsilon=(2 \sigma)^{2} / \beta$, where $2.45 \sigma$ (for two-dimensional Gaussian) is the distance between the $95 \%$-edge and the centre, and $\beta$ is the value of the betatron-function (horizontal or vertical) at the scraper position.

For acceptance measurements, an rf signal is used to "blow-up" the beam horizontally or vertically. If this is done until a pre-selected decrease in beam current, one is assured that the beam fills the entire acceptance of the machine. Again, the scraper blade is moved in and the remaining beam current decreases quadratically with the blade position in the vicinity of the beam edge. This is used to determine the exact position of the beam edge by fitting a parabola to the measured points around the edge. The acceptance is calculated as $\left(X_{\text {edge }}-X_{\text {centre }}\right)^{2} / \beta$, where $X_{\text {edge }}$ and $X_{\text {centre }}$ are the respective positions at the beam edge and centre.

\section{TUNE MEASUREMENTS}

It is desirable to measure the machine tunes in real time during the deceleration cycle, both at the plateaux and during ramps. The low intensity of the antiproton beam in the $\mathrm{AD}$ prevents the use of standard transverse Schottky techniques to measure the tunes using the new resonant Schottky pickup shown in Fig. 4. Therefore, a Beam Transfer Function (BTF) technique with the excitation of sidebands is necessary. For AD operation to date, tune measurements on different momentum plateaux are carried out using a swept BTF method and a network analyzer. This technique is slow due to pauses required on the plateaux, causes beam blow-up and is not applicable to measurements on the ramps. Hence, an improved system is under development [7]. This method uses an Mshaped power spectrum to excite the beam in a band around the expected frequency of a betatron side band. Excitation at the betatron frequency, where the beam response is highest, is thus minimised and the measurement of the BTF, hence the tune, may be performed with much reduced emittance blow-up.

The receiving system consists of: (a) an electrostatic PU made resonant at $5.7 \mathrm{MHz}$ with a Q factor of 900 and (b) a low noise amplifier using feedback to simulate a "noise free" resistor that de-tunes the system to a $\mathrm{Q}$ of 3. The high $\mathrm{Q}$ of the PU is obtained by having the coil inside the vacuum chamber, as shown. This ensemble gives a bandwidth of $1 \mathrm{MHz}$, wide enough to always contain at least one betatron side band, in which the Johnson noise from the losses in the coil is dominant. The system Q of 3 means that tuning of the PU is not needed. After signal conditioning to overcome the quantisation noise, the signals would be digitally down-converted and postprocessed in the same DRX board used for intensity evaluation [5]. In this manner, the DRX system will permit rapid measurements of tunes, intensity and other parameters in an integrated manner, i.e. all along the $\mathrm{AD}$ cycle, on the plateaux and on the ramps and using the same beam, without recourse to cycle pauses at different momenta as at present.

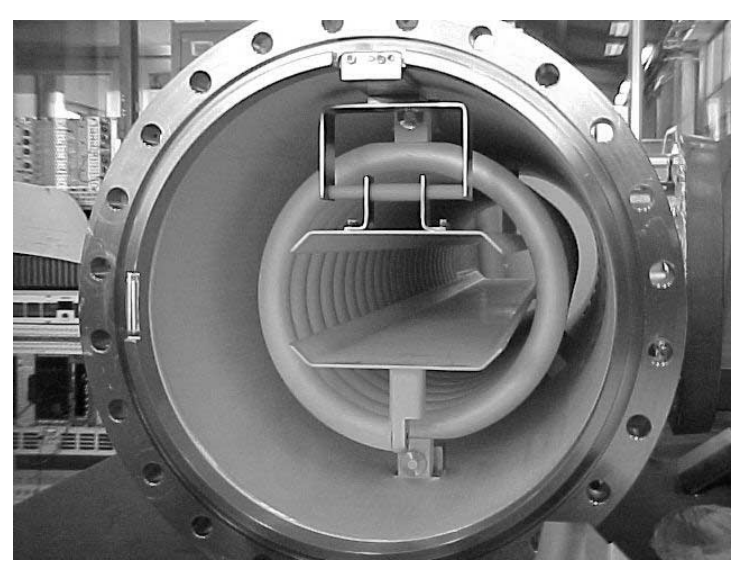

Figure 4: The Transverse Resonant "Schottky" PU.

\section{OTHER SYSTEMS}

The $\mathrm{AD}$ and the beam lines have other measurement systems installed for routine usage. There are 8 fast beam current transformers used for the production and test beams as well as for the extracted beam where feasible. There are 18 scintillation screens used for beam observation with TV cameras. A $100 \mathrm{MHz}$ set of digitizers perform measurement $\&$ correction of test beam coherent oscillations. Several Multi-wire Proportional Chambers provide beam profiles in the beam extraction lines. Special devices are used in the $60 \mathrm{keV}$ decelerating RFQ beam line.

\section{REFERENCES}

[1] S. Maury et al, Commissioning and Operation of the Antiproton Decelerator at CERN, this conference

[2] S. Baird et al., The antiproton decelerator: AD, Proc. of PAC 1997, Vancouver, Canada (1997).

[3 ]T. Eriksson et al for the AD Machine Team, Status Report on the AD, Proc.of LEAP 2000, Venezia, Italy.

[4] M.Le Gras, L.Soby, D. J. Williams, The closed orbit System for the AD, Proc. DIPAC 1999, Chester, UK.

[5] M.E.Angoletta et al, The new Digital Receiver based System for Antiprotons Beam Diagnostics, this conference.

[6] C. Gonzales, F. Pedersen, An ultra low-noise AC Beam transformer for deceleration and diagnostics of low intensity beams, Proc. of PAC 1999, NY, 1999.

[7] O.Marqversen et al, Real-time Tune Measurements on the CERN Antiproton Decelerator, Proc. DIPAC 2001 Grenoble, France. 